\title{
Existence and Global Exponential Stability of Positive Almost Periodic Solutions for a Time-Scales Model of Hematopoiesis with Multiple Time-Varying Variable Delays
}

\author{
K. Rajendra Prasad and Md. Khuddush \\ Andhra University \\ College of Science and Technology \\ Department of Applied Mathematics \\ Visakhapatnam, 530003, India
}

\begin{abstract}
In this paper we considered a hematopoiesis model with multiple time varying variable delays on time scales. By an application of fixed point theorem in a cone, sufficient conditions for the existence of unique positive almost periodic solution of the model are derived. We also investigated global exponential stability of the positive almost periodic solution of the model.
\end{abstract}

AMS Subject Classifications: 34C27, 34K20.

Keywords: Hematopoiesis model, time scales, almost periodic positive solution, fixed point theorem, cone, Gronwall inequality.

\section{Introduction}

The hematopoietic system is the best characterized model in stem cell biology. Hematopoietic stem cells are undifferentiated cells, located in the bone marrow, with unique abilities of differentiation i.e., production of cells committed to one blood cell lineage: neutrophils (white blood cells), erythrocytes (red blood cells) or megakaryocytes (platelets) and self-renewal (production of a cell with the same properties). These cells are at the root of the blood production process, called hematopoiesis. Through

Received May 8, 2019; Accepted August 28, 2019

Communicated by Martin Bohner 
successive differentiating divisions, they produce mature blood cells, that enter into the bloodstream.

To describe the dynamics of red blood cells production, in 1976 Lasota and WażewskaCzyżewska [14] studied the following functional differential equation,

$$
\frac{d u(t)}{d t}=-\gamma u(t)+\beta e^{-\alpha u(t-\tau)}
$$

as a model for the survival of red blood cells in an animal. Here $u(t)$ denotes the number of red blood cells at time $t, \gamma>0$ is the probability of death of a red blood cell, $\beta$ and $\alpha$ are positive constants related to the production of red blood cells per unit time, and $\tau$ is the (constant) time delay between the production of immature red blood cells and their maturation for release in circulating bloodstream. In 1977, Mackey and Glass [17] studied almost similar model arose from white blood cell production,

$$
\frac{d u(t)}{d t}=-\gamma u(t)+\beta \frac{u(t-\tau)}{1+u(t-\tau)^{n}} .
$$

Here $u(t)$ is the density of mature cells in blood circulation, the function $\beta \frac{u(t-\tau)}{1+u(t-\tau)^{n}}$ is the blood cell reproduction, the time lag $u(t-\tau)$ described the maturational phase before blood cells are released into circulation, the mortality rate $\gamma u(t)$ was assumed to be proportional to the circulation. Equation (1.2) was introduced to explain the oscillations in numbers of neutrophils observed in some cases of chronic myelogenous leukemia $[16,17]$.

The Lasota-Ważewska model (1.1) was derived from an age structured partial differential equation, and delay was a consequence of its integration, and on the other hand, the Mackey-Glass model (1.2) had been set up directly into a delay differential equation. The non-monotone nature of the nonlinearity of (1.2) brought a much richer behavior than the derived differential equation (1.1). Both approaches remain milestones in the sense that they are still used as references for new mathematical models and the development of delay differential equations. The corresponding discrete analogue of (1.1) takes the form

$$
u(n+1)-u(n)=-\gamma u(n)+\beta e^{\alpha u(n-\tau)} .
$$

The asymptotic behavior of (1.3) was studied by Chen and Yu [4]. The discrete analogy of (1.2) with variable coefficients,

$$
u(n+1)=(1-\delta(n)) u(n)+\frac{\beta(n)}{1+u^{m}(n-\tau)}
$$

was studied by Braverman and Saker [2]. They studied oscillation and nonoscillation about the positive periodic solutions of (1.4). The mathematical model for hematopoiesis 
with several delays,

$$
\frac{d u(t)}{d t}=-\gamma(t) u(t)+\sum_{i=1}^{K} \beta_{i}(t) \frac{u^{m}\left(t-\tau_{i}(t)\right)}{1+u^{n}\left(t-\tau_{i}(t)\right)}, m \geq 0
$$

where $\gamma_{i}, \beta_{i}, \tau_{i}: \mathbb{R} \rightarrow[0, \infty]$ are continuous functions for $i=1,2,3, \cdots, K$, is an appropriate extension of (1.2) was investigated for its qualitative properties in the papers [6-8, 15, 30, 32].

As we know, the variation of the environment plays an important role in many biological dynamical systems. In particular, the effects of a periodically varying environment are important for evolutionary theory as the selective forces on systems in a fluctuating environment differ from those in a stable environment. Thus, the assumption of periodicity of the parameters in the system incorporates the periodicity of the environment. In real world phenomenon, the environment varies due to the factors such as seasonal effects of weather, food supplies, mating habits and harvesting. However, if the various constituent components of the temporally nonuniform environment is with incommensurable (nonintegral multiples) periods, then one has to consider the environment to be almost periodic, since there is no a priori reason to expect the existence of periodic solutions. For this reason, the assumption of almost periodicity is more realistic and more general when we consider the effects of the environmental factors. In fact, several different periodic models have been studied for example (see [3,9-13, 18-20,22, 23, 25-27]).

The study of dynamical systems on time scales is now an active area of research. The theory of time scales has received a lot of attention which unifies and extends continuous and discrete analysis. In fact, both continuous and discrete systems are very important in implementation and applications. But it is not wort to study the dynamics for continuous and discrete systems independently. Therefore, it is significant to study on time scales which can unifies the continuous and discrete situations.

Motivated by the aforementioned reasons, in this paper, we concentrated on the generalized nonlinear hematopoiesis model with $N$ time varying variable delays on time scales,

$$
u^{\Delta}(t)=-\gamma(t) u(t)+\sum_{k=1}^{N} \beta_{k}(t) \frac{u^{n_{k}}\left(t-\tau_{k}(t)\right)}{1+u^{r}\left(t-\tau_{k}(t)\right)}, \quad t \in \mathbb{T},
$$

where $\mathbb{T}$ is a time scale, $0 \leq n_{k} \leq 1, r>0$ and $\gamma, \beta_{k}, \tau_{k}: \mathbb{T} \rightarrow(0,+\infty)$ are almost periodic functions. The corresponding continuous analogue of (1.6) was recently studied by Zhou and Yang [31] and established sufficient conditions for the existence of positive almost periodic solutions. In the literature, to the best of our knowledge, no contribution exists for the discrete analogue of (1.6). In this paper, we establish sufficient conditions for the existence of unique positive almost periodic solutions and investigate global exponential stability of the positive almost periodic solutions of (1.6).

The rest of the paper is organized as follows. In Section 2, we introduce some notations, definitions and lemmas which are useful for our main results. Sufficient con- 
ditions for the existence and global exponential stability of unique positive almost periodic solution of system (1.6) are established in Section 3 and Section 4 respectively. In Section 5, an example is given to illustrate the feasibility of our results. Finally, we draw a conclusion in Section 6.

\section{Preliminaries}

In this section, we introduce some definitions and state some preliminary results which are useful in the sequel.

Definition 2.1 (See [1]). A time scale $\mathbb{T}$ is a nonempty closed subset of the real numbers $\mathbb{R}$. $\mathbb{T}$ has the topology that it inherits from the real numbers with the standard topology. It follows that the jump operators $\sigma, \rho: \mathbb{T} \rightarrow \mathbb{T}$,

$$
\sigma(t)=\inf \{r \in \mathbb{T}: r>t\}, \quad \rho(t)=\sup \{r \in \mathbb{T}: r<t\}
$$

(supplemented by $\inf \emptyset:=\sup \mathbb{T}$ and $\sup \emptyset:=\inf \mathbb{T}$ ) are well defined. The point $t \in \mathbb{T}$ is left-dense, left-scattered, right-dense, right-scattered if $\rho(t)=t, \rho(t)<t, \sigma(t)=$ $t, \sigma(t)<t$, respectively. If $\mathbb{T}$ has right-scattered minimum $m$, define $\mathbb{T}_{k}=\mathbb{T}-\{m\}$; otherwise set $\mathbb{T}_{k}=\mathbb{T}$. If $\mathbb{T}$ has left-scattered maximum $M$, define $\mathbb{T}^{k}=\mathbb{T}-\{m\}$; otherwise let $\mathbb{T}^{k}=\mathbb{T}$.

Definition 2.2 (See [1]). By an interval time scale, we mean the intersection of a real interval with a given time scale. i.e., $[a, b]_{\mathbb{T}}=[a, b] \cap \mathbb{T}$. Similarly other intervals can be defined.

Definition 2.3 (See [1]). A function $f: \mathbb{T} \rightarrow \mathbb{R}$ is rd-continuous provided it is continuous at each right-dense point in $\mathbb{T}$ and has a left-sided limit at each left-dense point in $\mathbb{T}$. The set of rd-continuous functions $f: \mathbb{T} \rightarrow \mathbb{R}$ will be denoted by $C_{r d}(\mathbb{T})=C_{r d}(\mathbb{T}, \mathbb{R})$.

Definition 2.4 (See [1]). A function $p: \mathbb{T} \rightarrow \mathbb{R}$ is called regressive if $1+\mu(t) p(t) \neq 0$ for all $t \in \mathbb{T}^{k}$ If $p$ is regressive function, then the generalized exponential function $e_{p}$ is defined by

$$
e_{p}(t, s)=\exp \left\{\int_{s}^{t} \xi_{\mu(x)}(p(x)) \Delta x\right\}
$$

with the cylinder transformation

$$
\xi_{h}(z)=\left\{\begin{array}{cc}
\frac{\log (1+h z)}{h}, & h \neq 0, \\
z, & h=0 .
\end{array}\right.
$$

Definition 2.5 (See [1]). A function $p: \mathbb{T} \rightarrow \mathbb{R}$ is called regressive provided $1+$ $\mu(t) p(t) \neq 0$ for all $t \in \mathbb{T}^{k} ; p: \mathbb{T} \rightarrow \mathbb{R}$ is called positively regressive provided $1+\mu(t) p(t)>0$ for all $t \in \mathbb{T}^{k}$ The set of all regressive and rd-continuous functions $p: \mathbb{T} \rightarrow \mathbb{R}$ will be denoted by $\mathcal{R}=\mathcal{R}(\mathbb{T}, \mathcal{R})$ and the set of all positively regressive functions and rd-continuous functions will be denoted by $\mathcal{R}^{+}=\mathcal{R}^{+}(\mathbb{T}, \mathcal{R})$. 
Lemma 2.6 (See [1]). Assume that $p, q: \mathbb{T} \rightarrow \mathbb{R}$ are two regressive functions; then

(i) $e_{0}(t, s) \equiv 1$ and $e_{p}(t, t) \equiv 1$;

(ii) $e_{p}(t, s)=1 / e_{p}(s, t)=e_{\ominus p}(s, t)$;

(iii) $e_{p}(t, s) e_{p}(s, r)=e_{p}(t, r)$;

(iv) $\left(e_{p}(t, s)\right)^{\Delta}=p(t) e_{p}(t, s)$.

Lemma 2.7 (See [1]). Suppose that $p \in \mathcal{R}^{+}$, then

(i) $e_{p}(t, s)>0$ for all $t, s \in \mathbb{T}$;

(ii) if $p(t) \leq q(t)$ for all $t \geq s, t, s \in \mathbb{T}$, then $e_{p}(t, s) \leq e_{q}(t, s)$ for all $t \geq s$.

Lemma 2.8 (See [1]). If $p \in \mathcal{R}$ and $a, b, c \in \mathbb{T}$, then

$$
\begin{gathered}
{\left[e_{p}(c, \cdot)\right]^{\Delta}=-p\left[e_{p}(c, \cdot)\right]^{\sigma},} \\
\int_{a}^{b} p(t) e_{p}(c, \sigma(t)) \Delta t=e_{p}(c, a)-e_{p}(c, b) .
\end{gathered}
$$

Lemma 2.9 (See [1]). Let $p: \mathbb{T} \rightarrow \mathbb{R}$ be right-dense continuous and regressive, $a \in \mathbb{T}$ and $u_{a} \in \mathbb{R}$. Then the unique solution of the initial value problem

$$
\begin{aligned}
u^{\Delta}(t) & =p(t) u(t)+f(t), \\
u(a) & =u_{a}
\end{aligned}
$$

is given by

$$
u(t)=e_{p}(t, a) u_{a}+\int_{a}^{t} e_{p}(t, \sigma(s)) f(s) \Delta s .
$$

Definition 2.10 (See [21]). A time scale $\mathbb{T}$ is called an almost periodic time scale if

$$
\Pi:=\{\tau \in \mathbb{R}: t+\tau \in \mathbb{T}, \forall t \in \mathbb{T}\} \neq\{0\} .
$$

Definition 2.11 (See [21]). Let $\mathbb{T}$ be an almost periodic time scale. A function $f \in$ $C(\mathbb{T}, \mathbb{R})$ is said to be almost periodic on $\mathbb{T}$, if, for any $\varepsilon>0$, the set

$$
E(\varepsilon, f)=\{\tau \in \Pi:|f(t+\tau)-f(t)|<\varepsilon, \forall t \in \mathbb{T}\}
$$

is relatively dense in $\mathbb{T}$; that is, for any $\varepsilon>0$, there exists a constant $l(\varepsilon)>0$ such that each interval of length $l(\varepsilon)$ contains at least one $\tau \in E(\varepsilon, f)$ such that

$$
|f(t+\tau)-f(t)|<\varepsilon, \quad \forall t \in \mathbb{T} .
$$

The set $E(\varepsilon, f)$ is called the $\varepsilon$-translation number of $f(t)$. We denote the set of all such functions by $A P(\mathbb{T})$. 
Lemma 2.12 (See [21]). If $f \in C(\mathbb{T}, \mathbb{R})$ is an almost periodic function, then $f$ is bounded on $\mathbb{T}$.

Lemma 2.13 (See [21]). If $f, g \in C(\mathbb{T}, \mathbb{R})$ are almost periodic functions, then $f+g, f g$ are also almost periodic.

Lemma 2.14 (See [21]). If $f \in C(\mathbb{T}, \mathbb{R})$ is almost periodic, then $F(t)=\int_{0}^{t} f(s) \Delta s$ is almost periodic if and only if $F(t)$ is bounded.

Lemma 2.15 (See [21]). If $f \in C(\mathbb{T}, \mathbb{R})$ is almost periodic and $F(\cdot)$ is uniformly continuous on the value field of $f(t)$, then $F \circ f$ is almost periodic.

Definition 2.16 (See [29]). Let $x \in \mathbb{R}^{m}$ and $A(t)$ be an $m \times m$ rd-continuous matrix on $\mathbb{T}$; the linear system

$$
x^{\Delta}(t)=A(t) x(t), \quad t \in \mathbb{T},
$$

is said to admit an exponential dichotomy on $\mathbb{T}$ if there exist positive constants $k, \alpha$, projection $P$, and the fundamental solution matrix $x(t)$ of (2.1) satisfying

$$
\begin{gathered}
\left|x(t) P x^{-1}(\sigma(s))\right|_{0} \leq k e_{\ominus \alpha}(t, \sigma(s)), s, t \in \mathbb{T}, t \geq s, \\
\left|x(t)(I-P) x^{-1}(\sigma(s))\right|_{0} \leq k e_{\ominus \alpha}(\sigma(s), t), \quad s, t \in \mathbb{T}, t \leq s,
\end{gathered}
$$

where $|\cdot|_{0}$ is a matrix norm on $\mathbb{T}$; that is, if $A=\left(a_{i j}\right)_{m \times m}$, then we can take $|A|_{0}=$ $\left(\sum_{i=1}^{m} \sum_{j=1}^{m}\left|a_{i j}\right|^{2}\right)^{1 / 2}$.

Lemma 2.17 (See [21]). If the linear system (2.1) admits an exponential dichotomy, then the following system $x^{\Delta}(t)=A(t) x(t)+f(t), \quad t \in \mathbb{T}$, has a solution as follows:

$$
x(t)=\int_{-\infty}^{t} x(t) P x^{-1}(\sigma(s)) f(s) \Delta s-\int_{t}^{+\infty} x(t)(I-P) x^{-1}(\sigma(s)) f(s) \Delta s,
$$

where $x(t)$ is the fundamental solution matrix of (2.1).

Lemma 2.18 (See [21]). Let $A(t)$ be a regressive $n \times n$ matrix-valued function on $\mathbb{T}$. Let $t_{0} \in \mathbb{T}$ and $u_{0} \in \mathbb{R}^{n}$, then the initial value problem

$$
u^{\Delta}(t)=A(t) u(t), \quad u\left(t_{0}\right)=u_{0}
$$

has a unique solution $u(t)$ as follows

$$
u(t)=e_{A}\left(t, t_{0}\right) u_{0} .
$$


Lemma 2.19 (See [21]). Let $d_{i}(t)$ be a function on $\mathbb{T}$, where $d_{i}(t)>0,-d_{i}(t) \in \mathcal{R}^{+}$, for all $t \in \mathbb{T}$ and $\min _{1 \leq i \leq m}\left\{\inf _{t \in \mathbb{T}} d_{i}(t)\right\}>0$. Then the linear system

$$
x^{\Delta}(t)=\operatorname{diag}\left(-d_{1}(t),-d_{2}(t), \cdots,-d_{m}(t)\right) x(t)
$$

admits an exponential dichotomy on $\mathbb{T}$.

Definition 2.20 (See [5]). Let $X$ be a Banach space then a nonempty closed subset $P$ of $X$ is a cone if

(i) $u \in P, \lambda \leq 0$ implies $\lambda u \in P$;

(ii) $u \in P,-u \in P$ implies $u=0$.

Every cone $P \subset X$ induces an ordering in $X$, we define ' $\leq$ ' with respect to $P$ by $x \leq y$ if and only if $y-x \in P$.

Definition 2.21 (See [5]). A cone $P$ of $X$ is said to be normal if there exists a positive constant $\delta$ such that $\|x+y\| \geq \delta$ for any $x, y \in P,\|x\|=\|y\|=1$.

A cone $P$ is called a solid cone if the interior of $P$ is nonempty, i.e., $P^{0} \neq \emptyset$.

Definition 2.22 (See [5]). $P$ is a cone of $X$ and $A: P \rightarrow P$ is an operator. $A$ is called nondecreasing, if $\theta \leq x \leq y$ implies $A x \leq A y$.

For convenience, we introduce few notations and assumptions:

$\left(H_{1}\right)$ For any bounded function $f(t)$, we denote $f^{U}=\sup _{t \in \mathbb{T}} f(t), f^{L}=\inf _{t \in \mathbb{T}} f(t)$.

$\left(H_{2}\right)$ We assume that the bounded almost periodic functions $\gamma(t), \beta_{k}(t), \tau_{k}(t)$ satisfy $0<\gamma^{L} \leq \gamma(t) \leq \gamma^{U}, 0<\beta_{k}^{L} \leq \beta_{k}(t) \leq \beta_{k}^{U}, 0<\tau_{k}^{L} \leq \tau_{k}(t) \leq \tau_{k}^{U}$ for $k=1,2,3, \cdots, N$ and $-\gamma(t) \in \mathcal{R}^{+}$where $\mathcal{R}^{+}$is defined in the Definition 2.5.

$\left(H_{3}\right)$ Due to biological realistic of the model (1.6), we restrict our attention to positive solutions of equation (1.6).

$\left(H_{4}\right)$ The initial conditions associated with equation (1.6):

$$
u(t ; \varphi)=\varphi(t) \text { for } t \in\left[-\tau^{*}, 0\right]_{\mathbb{T}}, \tau^{*}=\max _{1 \leq k \leq N}\left\{\tau_{k}^{U}\right\}
$$

where $\varphi(\cdot)$ denotes a real valued bounded rd-continuous function defined on $\left[-\tau^{*}, 0\right]_{\mathbb{T}}$. 


\section{Existence of Unique Positive Almost Periodic Solu- tions}

In this section, we derive sufficient conditions for the existence of unique positive almost periodic solutions for the system (1.6).

Let $X=\{u: u \in A P(\mathbb{T})\}$ with norm $\|u\|=\sup _{t \in \mathbb{T}}|x(t)|$, then $X$ is Banach space. Let $P$ be a cone of $X$ defined by

$$
P=\{u \in X: u(t) \geq 0, t \in \mathbb{T}\},
$$

it is readily verified that $P$ is a normal solid cone of $X$. And interior of $P$ denotes by

$$
P^{0}=\{u \in P: \text { there exists } \varepsilon>0 \text { such that } u(t) \geq \varepsilon, t \in \mathbb{T}\} .
$$

In order to obtain existence of the unique positive almost periodic solution of (1.6), we will make use of the following fixed point theorem in a cone.

Theorem 3.1 (See [6]). Let $P$ be a normal and a solid cone in a real Banach space $X$, and let $\psi: P^{0} \rightarrow P^{0}$ be a nondecreasing operator, where $P^{0}$ is the interior of $P$. Suppose that there exists a function $\phi:(0,1) \times P^{0} \rightarrow(0, \infty)$ such that for each $\lambda \in(0,1)$ and $u \in P^{0}$,

(a) $\phi(\lambda, u)>\lambda$

(b) $\phi(\lambda, \cdot)$ is nondecreasing in $P^{0}$;

(c) $\psi(\lambda u) \geq \phi(\lambda, u) \psi(u)$.

And there exists $v \in P^{0}$ such that $\psi(v) \geq v$. Then $\psi$ has a unique fixed point $u^{*}$ in $P^{0}$. Moreover, given first term $u_{0} \in P^{0}$, the iterative sequence $u_{n}=\psi\left(u_{n-1}\right), n \in \mathbb{N}$ satisfies $\left\|u_{n}-u_{n}^{*}\right\| \rightarrow 0$ as $n \rightarrow \infty$.

Theorem 3.2. Assume that $0<r<n_{k}$. Then equation (1.6) has unique positive almost periodic solution $u^{*}$ with positive infimum provided $n_{k}<1$ or $n_{k}=1$ and $\sum_{k=1}^{N} \beta_{k}^{L}>\gamma^{U}$.

Proof. Define the operator $\psi: P^{0} \rightarrow P^{0}$ by

$$
\psi(u)(t)=\int_{-\infty}^{t} e_{-\gamma}(t, \sigma(s)) \sum_{k=1}^{N} \beta_{k}(s) \frac{u^{n_{k}}\left(s-\tau_{k}(s)\right)}{1+u^{r}\left(s-\tau_{k}(s)\right)} \Delta s, \quad t \in \mathbb{T} .
$$

Since $r<n_{k}$, the function $\frac{u^{n_{k}}}{1+u^{r}}$ is nondecreasing on $u \in(0,+\infty)$, so $\psi$ is nondecreasing operator. Let $\bar{u} \in P^{0}$. Then, it follows from Lemmas 2.12-2.15 that $\psi(\bar{u}) \in$ 
$A P(\mathbb{T})$. In addition, there exists $\varepsilon>0$ such that $\bar{u}(t) \geq \varepsilon$ for all $t \in \mathbb{T}$. So

$$
\begin{aligned}
\psi(\bar{u})(t) & \geq \int_{-\infty}^{t} e_{-\gamma^{U}}(t, \sigma(s)) \sum_{k=1}^{N} \beta_{k}^{L} \frac{\varepsilon^{n_{k}}}{1+\varepsilon^{r}} \Delta s \\
& =\frac{1}{\gamma^{U}\left(1+\varepsilon^{r}\right)} \sum_{k=1}^{N} \beta_{k}^{L} \varepsilon^{n_{k}}>0,
\end{aligned}
$$

for all $t \in \mathbb{T}$, implies that $\psi(\bar{u}) \in P^{0}$. Thus, $\psi$ is self map on $P^{0}$. Let

$$
\theta_{k}(\lambda, u)=\lambda^{n_{k}} \frac{1+u^{r}}{1+\lambda^{r} u^{r}}, \quad \lambda \in(0,1), \quad u>0 .
$$

Then $\theta_{k}(\lambda, \cdot)$ is nondecreasing in $u \in(0,+\infty)$ and $\theta_{k}(\lambda, u)>\lambda$ for each $\lambda \in(0,1)$ and $u>0$. Next, define the mapping $\phi(\lambda, u)$ by

$$
\phi(\lambda, u)=\min _{1 \leq k \leq N} \theta_{k}\left(\lambda, \inf _{t \in \mathbb{T}} u(t)\right) .
$$

Then,

$$
\begin{aligned}
& \psi(\lambda u)(t)= \int_{-\infty}^{t} e_{-\gamma}(t, \sigma(s)) \sum_{k=1}^{N} \beta_{k}(s) \frac{\lambda^{n_{k}} u^{n_{k}}\left(s-\tau_{k}(s)\right)}{1+\lambda^{r} u^{r}\left(s-\tau_{k}(s)\right)} \Delta s \\
&= \int_{-\infty}^{t} e_{-\gamma}(t, \sigma(s)) \sum_{k=1}^{N} \beta_{k}(s) \frac{u^{n_{k}}\left(s-\tau_{k}(s)\right)}{1+u^{r}\left(s-\tau_{k}(s)\right)} \times \\
& \frac{\lambda^{n_{k}}\left(1+u^{r}\left(s-\tau_{k}(s)\right)\right)}{1+\lambda^{r} u^{r}\left(s-\tau_{k}(s)\right)} \Delta s \\
& \geq \phi(\lambda, u) \int_{-\infty}^{t} e_{-\gamma}(t, \sigma(s)) \sum_{k=1}^{N} \beta_{k}(s) \frac{u^{n_{k}}\left(s-\tau_{k}(s)\right)}{1+u^{r}\left(s-\tau_{k}(s)\right)} \Delta s \\
&=\phi(\lambda, u) \psi(u)(t),
\end{aligned}
$$

$\phi(\lambda, u)>\lambda$ and $\phi(\lambda, \cdot)$ is nondecreasing in $P^{0}$ for all $\lambda \in(0,1)$ and $u \in P^{0}$. Further, either $n_{k}<1$ or $n_{k}=1$ and $\sum_{k=1}^{N} \beta_{k}^{L}>\gamma^{U}$, we can choose sufficiently small $\varepsilon>0$ such that

$$
\frac{1}{\gamma^{U}} \sum_{k=1}^{N} \beta_{k}^{L} \frac{\varepsilon^{n_{k}}}{1+\varepsilon^{r}} \geq \varepsilon
$$

That is,

$$
\psi(\varepsilon)(t) \geq \frac{1}{\gamma^{U}} \sum_{k=1}^{N} \beta_{k}^{L} \frac{\varepsilon^{n_{k}}}{1+\varepsilon^{r}} \geq \varepsilon
$$


for all $t \in \mathbb{T}$. Therefore, all the assumptions of Theorem 3.1 are satisfied. Thus, $\psi$ has a unique fixed point $u^{*}>0$, i.e., equation (1.6) has a unique positive almost periodic solution $u^{*}(t)$. Moreover, $\left\|u_{n}-u^{*}\right\| \rightarrow 0,(n \rightarrow \infty), u_{n}=\psi\left(u_{n-1}\right)(n=1,2,3, \cdots)$ for any initial $u_{0} \in P^{0}$. This completes proof.

Theorem 3.3. Assume that $0<n_{k}<r, 0<n_{k} \leq 1$ and

$$
\frac{1}{\gamma^{L}} \sum_{k=1}^{N} \beta_{k}^{U} \xi_{k} \leq\left(\frac{n_{j}}{r-n_{j}}\right)^{1 / r} \text { for } 1 \leq j \leq N, \text { where } \xi_{k}=\frac{\left(\frac{n_{k}}{r-n_{k}}\right)^{n_{k} / r}}{1+\frac{n_{k}}{r-n_{k}}}
$$

Then (1.6) has unique positive almost periodic solution $u^{*}$ with positive infimum provided that $n_{k}<1$ or $n_{k}=1$ and $\sum_{k=1}^{N} \beta_{k}^{L}>\gamma^{U}$.

Proof. Denote $\delta=\min _{1 \leq k \leq N}\left(\frac{n_{k}}{r-n_{k}}\right)^{1 / r}, \xi_{k}=\frac{\delta^{n_{k}}}{1+\delta^{r}}$ and letting

$$
f(u)=\left\{\begin{array}{cl}
\frac{u^{n_{k}}}{1+u^{r}}, & u \leq \delta, \\
\xi_{k}, & u>\delta .
\end{array}\right.
$$

Firstly, we show that (1.6) has an almost periodic solution $\bar{u}(t)$ with positive infimum, which is expressed as

$$
\bar{u}(t)=\int_{-\infty}^{t} e_{-\gamma}(t, \sigma(s)) \sum_{k=1}^{N} \beta_{k}(s) f\left[\bar{u}\left(s-\tau_{k}(s)\right)\right] \Delta s, \quad t \in \mathbb{T} .
$$

Let $\omega$ be a nonnegative almost periodic solution of (1.6). Since every $\tau_{k}$ is almost periodic, it follows that $\omega\left(\cdot-\tau_{k}(\cdot)\right)$ is also almost periodic for $k=1,2,3, \cdots, N$. Consequently, $\beta_{k}(\cdot) \frac{\omega^{n_{k}}\left(\cdot-\tau_{k}(\cdot)\right)}{1+\omega^{r}\left(\cdot-\tau_{k}(\cdot)\right)} \in A P(\mathbb{T}), k=1,2,3, \cdots, N$. Since $\gamma^{U}>0$, using Lemmas 2.17-2.19, we get

$$
\omega(t)=\int_{-\infty}^{t} e_{-\gamma}(t, \sigma(s)) \sum_{k=1}^{N} \beta_{k}(s) \frac{\omega^{n_{k}}\left(s-\tau_{k}(s)\right)}{1+\omega^{r}\left(s-\tau_{k}(s)\right)} \Delta s, \quad t \in \mathbb{T} .
$$

Using the hypothesis and $\sup _{u>0} \frac{u^{n_{k}}}{1+u^{r}}=\xi_{k}$, for all $t \in \mathbb{T}$, we have

$$
\begin{aligned}
\omega(t) & \leq \int_{-\infty}^{t} e_{-\gamma^{L}}(t, \sigma(s)) \sum_{k=1}^{N} \beta_{k}^{U} \xi_{k} \Delta s \\
& =\frac{1}{\gamma^{L}} \sum_{k=1}^{N} \beta_{k}^{U} \xi_{k}<\delta .
\end{aligned}
$$


From (3.1) and (3.3), we can conclude that

$$
\frac{\omega^{n_{k}}\left(s-\tau_{k}(s)\right)}{1+\omega^{r}\left(s-\tau_{k}(s)\right)}=f\left[\omega\left(s-\tau_{k}(s)\right)\right], \quad s \in \mathbb{T} .
$$

Therefore,

$$
\omega(t)=\int_{-\infty}^{t} e_{-\gamma}(t, \sigma(s)) \sum_{k=1}^{N} \beta_{k}(s) f\left[\omega\left(s-\tau_{k}(s)\right)\right] \Delta s, \quad t \in \mathbb{T},
$$

is an almost periodic solution of (1.6) with positive infimum, and vice versa. Define a nonlinear operator

$$
\kappa(u)(t)=\int_{-\infty}^{t} e_{-\gamma}(t, \sigma(s)) \sum_{k=1}^{N} \beta_{k}(s) f\left[u\left(s-\tau_{k}(s)\right)\right] \Delta s, \quad t \in \mathbb{T} .
$$

By direct calculation, we can conclude that $\kappa$ is a nondecreasing operator on $P^{0}$. Similar to the corresponding proof in Theorem 3.2, we can also show that $\kappa$ maps from $P^{0}$ to $P^{0}$. Also for $\lambda \in(0,1)$ and $u>0$,

$$
\sigma_{k}(\lambda, u):=\frac{f(\lambda u)}{f(u)}=\left\{\begin{array}{cl}
1, & u \geq \frac{\delta}{\lambda}, \\
\left(\frac{\lambda^{n_{k}} u^{n_{k}}}{1+\lambda^{r} u^{r}}\right) \frac{1}{\xi_{k}}, & \delta<u<\frac{\delta}{\lambda}, \\
\theta_{k}(\lambda, u), & u \leq \delta,
\end{array}\right.
$$

where $\theta_{k}(\lambda, u)$ be the same as in the proof of Theorem 3.2, and $\sigma_{k}(\lambda, \cdot)$ is nondecreasing on $(0, \infty)$. In addition, in the case of $u \geq \frac{\delta}{\lambda}$ and $u \leq \delta$, it is evident that $\sigma_{k}(\lambda, u)>\lambda$; in the case of $\delta<u<\frac{\delta}{\lambda}$, by simple calculation, we obtain $\sigma_{k}(\lambda, u)>\lambda^{n_{k}} \geq \lambda$. Define the mapping $\mathcal{F}(\lambda, u)$ by

$$
\mathcal{F}(\lambda, u)=\min _{1 \leq k \leq N} \sigma_{k}\left(\lambda, \inf _{t \in \mathbb{T}} u(t)\right), \quad \lambda \in(0,1), \quad u \in P^{0}
$$


Then, we get

$$
\begin{aligned}
& \kappa(\lambda u)(t)= \int_{-\infty}^{t} e_{-\gamma}(t, \sigma(s)) \sum_{k=1}^{N} \beta_{k}(s) \frac{\lambda^{n_{k}} u^{n_{k}}\left(s-\tau_{k}(s)\right)}{1+\lambda^{r} u^{r}\left(s-\tau_{k}(s)\right)} \Delta s \\
&= \int_{-\infty}^{t} e_{-\gamma}(t, \sigma(s)) \sum_{k=1}^{N} \beta_{k}(s) \frac{u^{n_{k}}\left(s-\tau_{k}(s)\right)}{1+u^{r}\left(s-\tau_{k}(s)\right)} \times \\
& \frac{\lambda^{n_{k}}\left(1+u^{r}\left(s-\tau_{k}(s)\right)\right)}{1+\lambda^{r} u^{r}\left(s-\tau_{k}(s)\right)} \Delta s \\
& \geq \mathcal{F}(\lambda, u) \int_{-\infty}^{t} e_{-\gamma}(t, \sigma(s)) \sum_{k=1}^{N} \beta_{k}(s) \frac{u^{n_{k}}\left(s-\tau_{k}(s)\right)}{1+u^{r}\left(s-\tau_{k}(s)\right)} \Delta s \\
&=\mathcal{F}(\lambda, u) \kappa(u)(t),
\end{aligned}
$$

$\mathcal{F}(\lambda, u)>\lambda$ and $\mathcal{F}(\lambda, \cdot)$ is nondecreasing in $P^{0}$ for all $\lambda \in(0,1)$ and $u \in P^{0}$. Rest of the proof is similar to that of Theorem 3.2. So we omit the details. This completes the proof.

Remark 3.4. For the case $n_{k}=0$ and $r>0$, the results established in [28].

\section{Stability Analysis}

In this section, we investigate the global exponential stability of the positive almost periodic solution for (1.6) with $n_{k}=1$. We recall the Gronwall inequality on time scales, which can be found in [1].

Theorem 4.1 (Gronwall inequality). Let $y \in C_{r d}, p \in \mathcal{R}^{+}, p \geq 0$ and $k \in \mathbb{R}$. Then

$$
y(t) \leq k+\int_{t_{0}}^{t} y(s) p(s) d s \text { for all } t \in \mathbb{T}
$$

implies

$$
y(t) \leq k e_{p}\left(t, t_{0}\right) \text { for all } t \in \mathbb{T} .
$$

In the next theorem, we use following notations: Let $u(t)$ be the positive almost periodic solution for (1.6) and $w^{*}(t)$ be an arbitrary solution of the equation (1.6) satisfying

$$
0<\liminf _{t \rightarrow \infty} w^{*}(t) \leq \limsup _{t \rightarrow \infty} w^{*}(t)<+\infty .
$$

Since $\tau_{k}$ is a bounded almost periodic function, there exists a sufficiently large $t_{0}>0$ such that

$$
a_{k}:=\min \left\{\inf _{t \in\left[t_{0},+\infty\right)_{\mathbb{T}}} u\left(t-\tau_{k}(t)\right), \inf _{t \in\left[t_{0},+\infty\right)_{\mathbb{T}}} w^{*}\left(t-\tau_{k}(t)\right)\right\}>0
$$


and

$$
b_{k}:=\max \left\{\sup _{t \in\left[t_{0},+\infty\right)_{\mathbb{T}}} u\left(t-\tau_{k}(t)\right), \sup _{t \in\left[t_{0},+\infty\right)_{\mathbb{T}}} w^{*}\left(t-\tau_{k}(t)\right)\right\}<+\infty .
$$

By the mean value theorem, it is not difficult to prove that

$$
\left|\frac{u\left(t-\tau_{k}(t)\right)}{1+u^{r}\left(t-\tau_{k}(t)\right)}-\frac{w\left(t-\tau_{k}(t)\right)}{1+w^{* r}\left(t-\tau_{k}(t)\right)}\right| \leq \mathcal{L}\left|u\left(t-\tau_{k}(t)\right)-w^{*}\left(t-\tau_{k}(t)\right)\right|
$$

where

$$
\mathcal{L}:=\max _{x \in\left[a_{k}, b_{k}\right]}\left\{\frac{1+|1-r| x^{r}}{\left(1+x^{r}\right)^{2}}\right\}
$$

Theorem 4.2. Assume that $\gamma^{L}>\eta$ where $\eta=\sup _{t \in\left[t_{0}, \infty\right)_{\mathbb{T}}} \frac{\mathcal{L} \sum_{k=1}^{N} \beta_{k}^{U}}{1-\mu(t) \gamma(t)}$ and $n_{k}=1$. Then the unique positive almost periodic solution of (1.6) is globally exponentially stable.

Proof. Let $w^{*}$ be a unique positive almost periodic solution for (1.6). Let $\phi(t)$ be the initial function of $w^{*}, w^{*}(t ; \phi)=\phi(t)$ for $t \in\left[-\tau^{*}, 0\right]_{\mathbb{T}}$. Now we prove $w^{*}(t)$ is globally exponentially stable.

Suppose $u(t)$ is arbitrary solution of equation (1.6) with initial function $u(t ; \varphi)=$ $\varphi(t)>0, t \in\left[-\tau^{*}, 0\right]_{\mathbb{T}}$. Let $v(t)=u(t)-w^{*}(t)$, then we have

$$
\begin{aligned}
v^{\Delta}(t)=\left(u(t)-w^{*}(t)\right)^{\Delta} & \\
=-\gamma(t)\left(u(t)-w^{*}(t)\right)+\sum_{k=1}^{N} \beta_{k}(t) & {\left[\frac{u\left(t-\tau_{k}(t)\right)}{1+u^{r}\left(t-\tau_{k}(t)\right)}\right.} \\
& \left.-\frac{w^{*}\left(t-\tau_{k}(t)\right)}{1+w^{* r}\left(t-\tau_{k}(t)\right)}\right] .
\end{aligned}
$$

Let

$$
r(t)=\sum_{k=1}^{N} \beta_{k}(t)\left[\frac{u\left(t-\tau_{k}(t)\right)}{1+u^{r}\left(t-\tau_{k}(t)\right)}-\frac{w^{*}\left(t-\tau_{k}(t)\right)}{1+w^{* r}\left(t-\tau_{k}(t)\right)}\right],
$$

then it follows from (4.1) that

$$
v^{\Delta}(t)=-\gamma(t) v(t)+r(t) .
$$

From Lemma 2.9, $v(t)$ can be expressed as

$$
\begin{aligned}
v(t) & =e_{-\gamma}\left(t, t_{0}\right) v\left(t_{0}\right)+\int_{t_{0}}^{t} e_{-\gamma}(t, \sigma(s)) r(s) \Delta s, \quad\left(t \geq t_{0}\right), \quad t_{0} \in\left[-\tau^{*}, 0\right]_{\mathbb{T}} \\
& =e_{-\gamma}\left(t, t_{0}\right) v\left(t_{0}\right)+\int_{t_{0}}^{t} \frac{1}{1-\mu(s) \gamma(s)} e_{-\gamma}(t, s) r(s) \Delta s
\end{aligned}
$$


Using initial functions, we obtain

$$
v(t)=e_{-\gamma}\left(t, t_{0}\right)\left(\varphi\left(t_{0}\right)-\phi\left(t_{0}\right)\right)+\int_{t_{0}}^{t} \frac{1}{1-\mu(s) \gamma(s)} e_{-\gamma}(t, s) r(s) \Delta s .
$$

Note that, by the mean value theorem,

$$
\begin{aligned}
|r(t)| & \leq \sum_{k=1}^{N} \beta_{k}(t)\left|\frac{u\left(t-\tau_{k}(t)\right)}{1+u^{r}\left(t-\tau_{k}(t)\right)}-\frac{w^{*}\left(t-\tau_{k}(t)\right)}{1+w^{* r}\left(t-\tau_{k}(t)\right)}\right| \\
& \leq \sum_{k=1}^{N} \beta_{k}(t) \mathcal{L}\left|u\left(t-\tau_{k}(t)\right)-w^{*}\left(t-\tau_{k}(t)\right)\right| \\
& \leq\left\|u-w^{*}\right\| \mathcal{L} \sum_{k=1}^{N} \beta_{k}^{U} .
\end{aligned}
$$

So that

$$
\|r(t)\| \leq\left\|u-w^{*}\right\| \mathcal{L} \sum_{k=1}^{N} \beta_{k}^{U}=\|v\| \mathcal{L} \sum_{k=1}^{N} \beta_{k}^{U} .
$$

Taking norm on both sides of (4.2) and using (4.4), we obtain

$$
\begin{aligned}
\|v(t)\| & \leq e_{-\gamma}\left(t, t_{0}\right)\|\varphi-\phi\|+\int_{t_{0}}^{t} \frac{e_{-\gamma}(t, s)}{1-\mu(s) \gamma(s)}\|r(s)\| \Delta s \\
& \leq e_{-\gamma}\left(t, t_{0}\right)\|\varphi-\phi\|+\int_{t_{0}}^{t} \frac{e_{-\gamma}(t, s)}{1-\mu(s) \gamma(s)}\|v\| \mathcal{L} \sum_{k=1}^{N} \beta_{k}^{U} \Delta s .
\end{aligned}
$$

From (4.5) and Lemma 2.6, we have

$$
\frac{\|v(t)\|}{e_{-\gamma}\left(t, t_{0}\right)} \leq\|\varphi-\phi\|+\int_{t_{0}}^{t} \frac{\|v\|}{e_{-\gamma}\left(s, t_{0}\right)} \frac{\mathcal{L} \sum_{k=1}^{N} \beta_{k}^{U}}{1-\mu(s) \gamma(s)} \Delta s .
$$

By the Gronwall inequality, we get

$$
\frac{\|v(t)\|}{e_{-\gamma}\left(t, t_{0}\right)} \leq\|\varphi-\phi\| e_{\eta}\left(t, t_{0}\right)
$$

which implies that

$$
\begin{aligned}
\|v(t)\| & \leq\|\varphi-\phi\| e_{\eta}\left(t, t_{0}\right) e_{-\gamma}\left(t, t_{0}\right) \\
& \leq\|\varphi-\phi\| e_{\eta}\left(t, t_{0}\right) e_{-\gamma^{L}}\left(t, t_{0}\right) \\
& \leq\|\varphi-\phi\| e_{-\left(\gamma^{L}-\eta\right)}\left(t, t_{0}\right) .
\end{aligned}
$$

That is $\left\|u(t)-w^{*}(t)\right\| \leq\|\varphi-\phi\| e_{-\left(\gamma^{L}-\eta\right)}\left(t, t_{0}\right), \gamma^{L}>\eta$, which means that $w^{*}(t)$ is globally exponentially stable. The proof is complete. 
Positive Almost Periodic Solutions for a Hematopoiesis Model

\section{Examples}

In this section, we give a few examples to illustrate our results.

Example 5.1. Consider the following model of hematopoiesis with multiple time varying variable delays described by

$$
\begin{aligned}
u^{\Delta}(t)=-6.9 u(t) & +\left(6+\frac{1}{2}|\cos \sqrt{7} t|\right) \times \frac{u^{1 / 3}\left(t-2 e^{\cos t}\right)}{1+u^{8 / 17}\left(t-2 e^{\cos t}\right)} \\
& +\left(2+\frac{1}{2}|\sin \sqrt{5} t|\right) \times \frac{u^{1 / 5}\left(t-2 e^{\cos t}\right)}{1+u^{8 / 17}\left(t-2 e^{\cos t}\right)}
\end{aligned}
$$

It is easy to check all the conditions of Theorem 3.2 are satisfied. Therefore, there exists a unique positive almost periodic solution for (5.1).

Example 5.2. The second example we consider is the following model of hematopoiesis with multiple time varying variable delays described by

$$
\begin{aligned}
u^{\Delta}(t)=-4 u(t) & +\left(3+\frac{1}{20}|\sin \sqrt{2} t|\right) \times \frac{u^{2 / 3}\left(t-\cos ^{2} \frac{t}{40}\right)}{1+u^{3 / 2}\left(t-\cos ^{2} \frac{t}{40}\right)} \\
& +\left(2+\frac{1}{10}|\cos \sqrt{5} t|\right) \times \frac{u^{3 / 4}\left(t-\cos ^{2} \frac{t}{40}\right)}{1+u^{3 / 2}\left(t-\cos ^{2} \frac{t}{40}\right)} .
\end{aligned}
$$

Here

$$
\gamma(t)=4, n_{1}=2 / 3, n_{2}=3 / 4, r=3 / 2 .
$$

We can calculate that,

$$
\begin{gathered}
\beta_{1}^{L}=3, \beta_{1}^{U}=3.05, \beta_{2}^{L}=2, \beta_{2}^{U}=2.1, \xi_{1} \approx 0.5031024, \xi_{2}=0.5 \\
\left(\frac{n_{1}}{r-n_{1}}\right)^{1 / r} \approx 0.8617739,\left(\frac{n_{2}}{r-n_{2}}\right)^{1 / r}=1, \frac{1}{\gamma^{L}} \sum_{k=1}^{N} \beta_{k}^{U} \xi_{k} \approx 0.6461156
\end{gathered}
$$

and hence

$$
\frac{1}{\gamma^{L}} \sum_{k=1}^{N} \beta_{k}^{U} \xi_{k} \leq\left(\frac{n_{j}}{r-n_{j}}\right)^{1 / r}, j=1,2 .
$$

This implies that all the assumptions of Theorem 3.3 are satisfied. Therefore, there exists a unique positive almost periodic solution for (5.2).

Example 5.3. In this example, we show global exponential stability of the following hematopoiesis model with time varying variable delays

$$
\begin{aligned}
u^{\Delta}(t)=-0.6 u(t) & +\left(\frac{1}{4}+\frac{1}{2}|\sin \sqrt{2} t|\right) \times \frac{u\left(t-2 e^{\sin t}\right)}{1+u^{65}\left(t-2 e^{\sin t}\right)} \\
+ & \left(\frac{1}{2}+|\cos \sqrt{3} t|\right) \times \frac{u\left(t-2 e^{\sin t}\right)}{1+u^{65}\left(t-2 e^{\sin t}\right)} .
\end{aligned}
$$


Here

$$
\gamma(t)=0.6, r=65, \beta_{1}(t)=\frac{1}{4}+\frac{1}{2}|\sin \sqrt{2} t|, \beta_{2}(t)=\frac{1}{2}+|\cos \sqrt{3} t| .
$$

On simple calculations, we get

$$
\beta_{1}^{L}=\frac{1}{4}, \beta_{1}^{U}=\frac{3}{4}, \beta_{2}^{L}=\frac{1}{2}, \beta_{2}^{U}=\frac{3}{2}, \mathcal{L} \leq 16.2539683
$$

If $\mathbb{T}=\mathbb{R}$, then $\mu(t)=0$ and hence

$$
\eta=\mathcal{L} \sum_{k=1}^{2} \beta_{k}^{U} \leq 0.1384278 \leq \gamma^{L}
$$

If $\mathbb{T}=\mathbb{Z}$, then $\mu(t)=1$ and hence

$$
\eta=\sup _{t \in\left[t_{0}, \infty\right)_{\mathbb{T}}} \frac{\mathcal{L} \sum_{k=1}^{2} \beta_{k}^{U}}{1-\gamma(t)} \leq 0.3460693 \leq \gamma^{L} .
$$

This implies that (5.3) satisfies the assumptions of Theorem 4.2. Therefore, equation (5.3) has a unique positive almost periodic solution $w^{*}(t)$, which is globally exponentially stable. The numerical simulations in Figure 5.1 strongly support the conclusion.

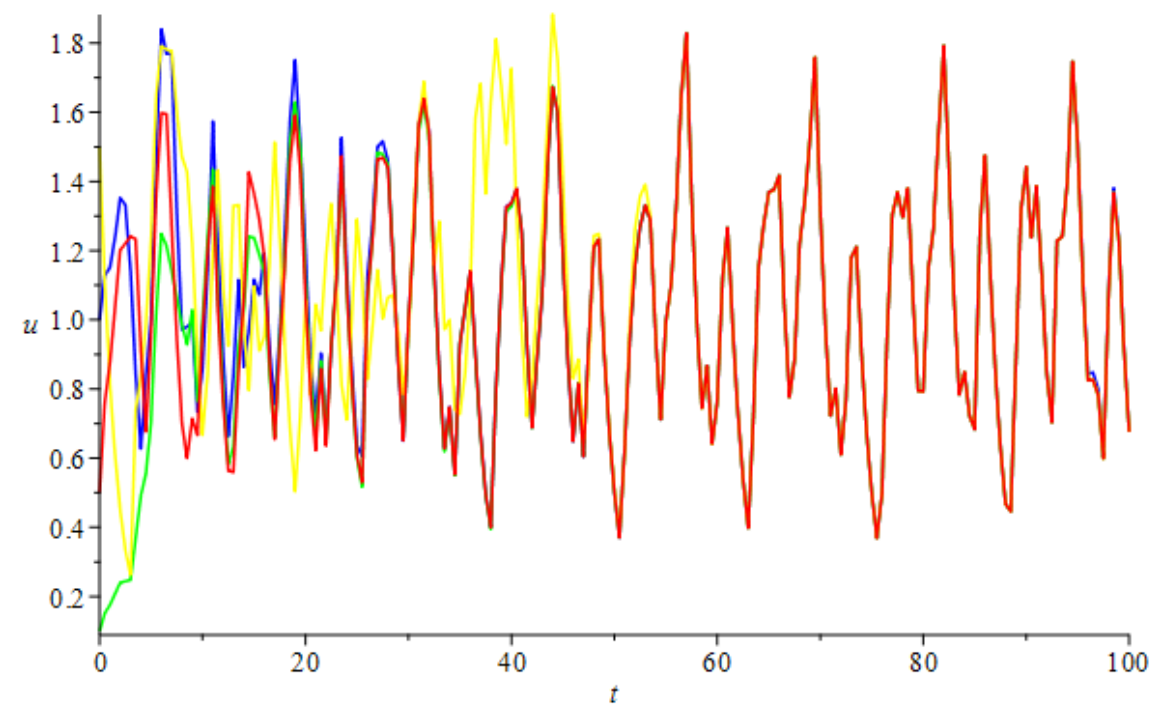

Figure 5.1: Numerical solution $u(t)$ of equation (5.3) for initial value $\varphi(s)=0.1,0.5$, $1,1.5 s \in[-2 e, 0]$. 


\section{Conclusion}

In the introduction section, we mentioned that one of our objectives is to unify the existence and stability of positive almost periodic solution for some differential equation and their corresponding discrete analogues. If $\mathbb{T}=\mathbb{R}$ and $\mathbb{T}=\mathbb{Z}$, then equation (1.6) reduces to

$$
u^{\prime}(t)=-\gamma(t) u(t)+\sum_{k=1}^{N} \beta_{k}(t) \frac{u^{n_{k}}\left(t-\tau_{k}(t)\right)}{1+u^{r}\left(t-\tau_{k}(t)\right)}, t \in \mathbb{R}
$$

and

$$
u(t+1)-u(t)=-\gamma(t) u(t)+\sum_{k=1}^{N} \beta_{k}(t) \frac{u^{n_{k}}\left(t-\tau_{k}(t)\right)}{1+u^{r}\left(t-\tau_{k}(t)\right)}, t \in \mathbb{Z}
$$

respectively. The existence of positive almost periodic solutions for more general models of hematopoiesis and its qualitative analysis is interesting one, which can be carried out in future.

\section{Acknowledgments}

The authors thank the referees for valuable suggestions and one of the authors $(\mathrm{Md}$. Khuddush) thanks UGC, New Delhi, Government of India for awarding SRF under MANF.

\section{References}

[1] M. Bohner, A. Peterson, Dynamic equations on time scales: An interoduction with Applications. Birkhäuser, Boston, (2001).

[2] E. Braverman, S. H. Saker, Permanence, oscillation and attractivity of the discrete hematopoiesis model with variable coefficients. Nonlinear Anal. 67 (2007) 29552965.

[3] Y. Chen, L. Huang, Existence and global attractivity of a positive periodic solution of a delayed periodic respiration model. Comput. Math. Appl. 49 (2005) 677-687.

[4] M. Chen, J. S. Yu, Oscillation and global Attractivity in the discrete LasotaWazewaska model. Soochow J. Math. 25 (1), 1-9, 1999.

[5] K. Deimling, Nonlinear functional analysis. Springer-Verlag, Berlin, 1985.

[6] T. Diagana, H. Zhou, Existence of positive almost periodic solutions to the hematopoiesis model. Appl. Math. Comput. 274 (2016) 644-648. 
[7] H. S. Ding, Q. L. Liu, J. J. Nieto, Existence of positive almost periodic solutions to a class of hematopoiesis model. Appl. Math. Model. 40 (2016) 3289-3297.

[8] H. S. Ding, H. M. N'Guerekata, J. J. Nieto, Weighted pseudo almost periodic solutions for a class of discrete hematopoiesis model. Rev. Mat. Complut. 26 (2013) 427-443.

[9] K. Gopalsamy, M. R. S. Kulenovic, G. Ladas, Environmental periodicity and time delays in a food limited population model. J. Math. Anal. Appl. 147 (1990) 545555 .

[10] K. Gopalsamy, S. Trofimchuk, Almost periodic solutions of Lasota-Wazewskatype delay differential equation. J. Math. Anal. Appl. 237 (1999) 106-127.

[11] J. R. Graef, C. Qian, P.W. Spikes, Oscillation and global attractivity in a periodic delay equation. Canad. Math. Bull. 38 (1996) 275-283.

[12] Y. Kuang, Delay Differential Equations with Applications in Population Dynamics. Academic Press, Boston, 1993.

[13] B. S. Laslli, B.G. Zhang, On a periodic delay population model. Quart. Appl. Math. 52 (1994) 35-42.

[14] A. Lasota, M. Ważewska-Czyżewska, Matematyczne problemy dynamiki ukladu krwinek czerwonych (Mathematical problems of the dynamics of red blood cell population), (in Polish). Matematyka Stosowana 6 (1976), 23-40.

[15] B. W. Liu, New results on the positive almost periodic solutions for a model of hematopoiesis. Nonlinear Anal. Real World Appl. 17 (2014) 252-264.

[16] J. Losson, M. C. Mackey, A. Longtin, Solution multistability in first order nonlinear differential delay equations. Chaos 3(2), 167-176, (1993).

[17] M. C. Mackey, L. Glass, Oscillation and chaos in physiological control systems. Science 197 (1977), 287-289.

[18] M. R. S. Kulenovic, G. Ladas, Y.G. Sficas, Global attractivity in population dynamics. Comput. Math. Appl. 18 (1989) 925-928.

[19] S. H. Saker, S. Agarwal, Oscillation and global attractivity in a nonlinear delay periodic model of respiratory dynamics. Comput. Math. Appl. 44 (2002) 623-632.

[20] S. H. Saker, S. Agarwal, Oscillation and global attractivity in a periodic Nicholson Blowflies Model. Math. Comput. Modelling 35 (2002) 719-731. 
[21] Y. Li, C. Wang, Uniformly almost periodic functions and almost periodic solutions to dynamic equations on time scales. Abstr. Appl. Anal. 2011, Art. ID 341520, 22 pp.

[22] A. Wan, D. Jiang, X. Xu, A new existence theory for positive periodic solutions to functional differential equations. Comput. Math. Appl. 47 (2004) 1257-1262.

[23] H. Wang, Positive periodic solutions of functional differential equations, J. Differential Equations 202 (2004) 354-366.

[24] P. X. Weng, Global attractivity of periodic solution in a model of hematopoiesis. Comput. Math. Appl. 44 (2002) 1019-1030.

[25] W. Xu, J. Li, Global attractivity of the model for the survival of red blood cells with several delays. Ann. Differential Equations 14 (1998) 357-363.

[26] J. Yan, A. Zhao, J. J. Nieto, Existence and global attractivity of positive periodic solution of periodic single-species impulsive Lotka-Volterra systems. Math. Comput. Modelling 40 (2004) 509-518.

[27] J. Yan, A. Zhao, W. Yan, Existence and global attractivity of periodic solution for an impulsive delay differential equation with Allee effect. J. Math. Anal. Appl. 309 (2005) 489-504.

[28] Z. Yao, Uniqueness and global exponential stability of almost periodic solution for Hematopoiesis model on time scales. J. Nonlinear. Sci. Appl. 8 (2015) 142-152.

[29] J. Zhang, M. Fan, Existence and roughness of exponential dichotomies of linear dynamic equations on time scales. Comput. Math. Appl. 59 (2010), no. 8, 26582675 .

[30] H. Zhang, M. Q. Yang, L. J. Wang, Existence and exponential convergence of the positive almost periodic solution for a model of hematopoiesis. Appl. Math. Lett. 26 (2013) 38-42.

[31] H. Zhou, L. Yang, A new result on the existence of positive almost periodic solution for generalized hematopoiesis model. J. Math. Anal. Appl. 462 (2018) 370379 .

[32] H. Zhou, W. Wang, Z. F. Zhou, Positive almost periodic solution for a model of hematopoiesis with infinite time delays and a nonlinear harvesting term. Abstr. Appl. Anal. (2013) 146729. 
